\title{
Oncolytic parvovirus for cancer of the brain: are we approaching human trials?
}
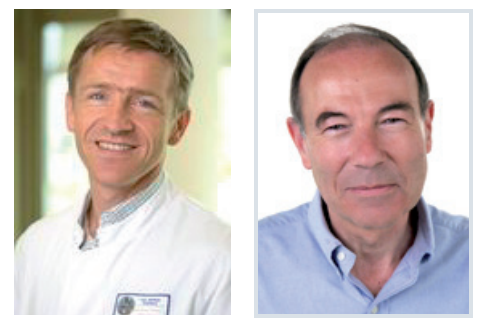

"It is most likely that the H-1PV trial will be the first trial of an oncolytic

virus in glioma patients that will use intratumoral and intravenous injections."

\section{Karsten Geletneky ${ }^{\dagger} \&$ Jean Rommelaere'}

'German Cancer Research Center, Department of Applied Tumor Virology, Im Neuenheimer Feld 242, 69120 Heidelberg, Germany

${ }^{\dagger}$ Author for correspondence: Department of Neurosurgery, University of Heidelberg, Im Neuenheimer Feld 400, 69120 Heidelberg, Germany = Tel.: +4962215639672 = kgeletneky@med.uni-heidelberg.de

The dismal prognosis of malignant glioma has prompted the ongoing evaluation of therapeutic alternatives other than the conventional strategies of surgery, radiation and cytoreductive chemotherapy [1]. One evolving field is the use of replication-competent oncolytic viruses (OV). The panel of viruses with oncolytic activity in tumor cells, in particular brain tumor cells, is quite diverse. It includes potentially pathogenic viruses that have to be genetically modified prior to therapeutic use, such as herpesvirus, adenovirus and poliovirus, or wild-type viruses, such as reovirus, vaccinia virus and Newcastle disease virus. These viruses represent the initial group of OVs that were investigated to treat brain tumors, but more viruses with promising antiglioma properties have recently been described, such as vesicular stomatitis virus, myxoma virus and Semliki Forest virus, which belong to the second wave of less commonly known candidates [2]. Along this line we started to investigate the oncolytic effects of a member of the parvovirus family, parvovirus $\mathrm{H}-1$ (H-1PV), on malignant gliomas.

\section{"After these initial therapeutic attempts, the translation of H-1PV from laboratory to clinical use lay dormant for more than 15 years."}

By origin, $\mathrm{H}-1 \mathrm{PV}$ is a small rat virus with a ssDNA genome of approximately 5000 bases that, at least under experimental conditions, is able to infect distinct species including humans [3]. Interestingly, the track record of antitumor effects by H-1PV infection, albeit not for brain tumors, is rather long: in the 1960s infected animals showed a clearly reduced rate of spontaneous development of tumors [4] and, in the 1980s, oncolytic effects induced by H-1PV infection of animals bearing solid tumors were reported [5]. In 1965, Toolan et al. injected H-1PV in two adolescent patients with osteosarcomas [6], since then, in the early 1990 s, H-1PV was administered in cutaneous metastases of 12 terminally ill cancer patients [7]. The H-1PV treatments, which by today's standards would not qualify as a clinical trial, were well tolerated but did not result in a significant tumor reduction. After these initial therapeutic attempts, the translation of H-1PV from laboratory to clinical use lay dormant for more than 15 years.

Based on the promising biological properties of H-1PV, we started to examine the potential of H-1PV for therapy of malignant gliomas. In the course of the experiments, even more favorable characteristics of H-1PV emerged, further supporting a possible clinical use. A short summary of the most important findings should illustrate the potential of the virus. H-1PV very efficiently infects and kills glioma cells in vitro, even at low virus doses [8]. In particular, a fact that was previously unknown is that $\mathrm{H}-1 \mathrm{PV}$-induced killing of glioma cells is independent from the resistance of cells to chemotherapy or the induction of apoptosis, which often limits therapeutic efficacy of current glioma therapies. Rather than inducing apoptosis, H-1PV triggers an alternative, autophagy-like death pathway [9]. Animal experiments substantiated the promising data from cell cultures. H-1PV infection of orthotopic experimental gliomas in rats resulted in complete regression of advanced and even symptomatic tumors without any side effects. Furthermore, almost unique for OVs, brain tumors disappeared not only after local virus therapy, which is the most common route of administration, but also after intravenous H-1PV injection [10].

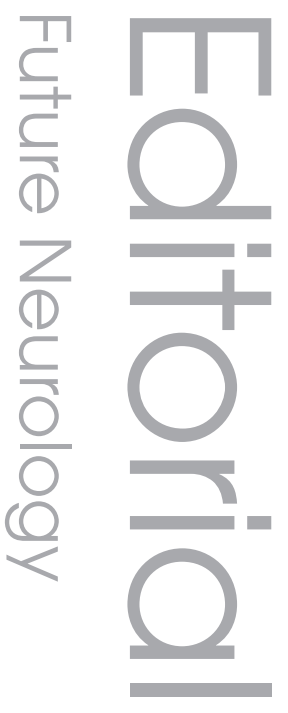

\section{Keywords}

- clinical trial $=$ malignant glioma $=$ oncolytic virus - parvovirus $\mathrm{H}-1$

\section{future Mredine $_{\text {Isg }}$}


Clearly, when compared with preclinical data on other OVs that have already been tested in clinical glioma trials, the H-1PV results look very powerful. However, even after obtaining such promising results there is a huge gap to bridge prior to using a new biological substance in patients. As trials of this kind are very expensive, the foremost requirement is funding. In the case of $\mathrm{H}-1 \mathrm{PV}$, the costs of all aspects of the intended Phase I/IIa trial will be sponsored by a private company, Oryx $\mathrm{GmbH}$ and Co. KG based in Munich, Germany. The next critical step towards a trial is the production of the virus according to Good Manufacturing Practice (GMP) standards, as it is required by regulatory authorities that only viruses of GMP grade are used in patients. This is no minor task as it is necessary to identify GMP facilities or companies that are capable of transforming the small-scale production of the virus for laboratory use to a GMP-compatible protocol, and to produce it at a much larger scale without compromising virus yields. For H-1PV, the establishment of the GMP process turned out to be a difficult task, and required close and continuous cooperation between the manufacturer and the scientific team, who finally succeeded in the production of virus stocks of clinical grade material. This clinical grade virus batch is currently undergoing extensive toxicology testing, which will hopefully confirm the anticipated purity and safety of H-1PV, even after repetitive administration at high doses. These results are still pending.

\footnotetext{
${ }^{66}$ Toxicology clearance of the Good Manufacturing Practice virus and federal approval of the modified trial concept are still pending."
}

Another crucial step towards clinical use is the generation of the trial protocol that needs to be approved by regulatory bodies. For Phase I/IIa trials, by definition, the foremost concern is to assess the safety profile of a new substance. However, it is of major interest to also generate data that address the question of antitumor activity, as well as more general questions, such as virus distribution and immunology other than safety-related immune effects. All these aspects have to be discussed with the authorities and be included in the final documentation. In previous meetings, the responsible federal agency, the Paul-Ehrlich Institute in Langen, Germany, had no general objections against a clinical trial in glioma patients; however, modifications of the initially suggested trial design were demanded and are currently being made. Although conclusive information about the protocol can only be given after final approval, it is most likely that the H-1PV trial will be the first trial of an $\mathrm{OV}$ in glioma patients that will use intratumoral and intravenous injections. This combinatory approach is intended to improve cerebral virus distribution to tumor cells remote from the main tumor bulk without compromising the opportunity to administer a high local dose.

In summary, it is becoming very likely that $\mathrm{H}-1 \mathrm{PV}$ will soon be investigated in a Phase I/IIa clinical trial in patients with malignant gliomas. Convincing preclinical data and successful production of clinical-grade material have already been achieved. Toxicology clearance of the GMP virus and federal approval of the modified trial concept are still pending.

\section{Acknowledgements}

The authors would like to thank all coworkers from the German Cancer Research Center, University of Heidelberg and Institut National de la Santé et de la Recherche Médicale, Heidelberg, Germany. The authors are grateful for the ongoing dedication and support from the sponsor, Oryx GmbH and Co KG.

\section{Financial \& competing interests disclosure}

This work is supported by Oryx GmbH and Co KG. The authors have no other relevant affliations or financial involvement with any organization or entity with a financial interest in or financial conflict with the subject matter or materials discussed in the manuscript apart from those disclosed.

No writing assistance was utilized in the production of this manuscript.

6. Toolan HW, Saunders EL Southam CM, Moore AE, Levin AG: $\mathrm{H}-1$ virus viremia in the human. Proc. Soc. Exp. Biol. Med. 119, 711-715 (1965)

7. Le Cesne A, Dupressoir T, Janin N et al. Intra-lesional administration of a live virus, parvovirus $\mathrm{H}-1$ (PVH-1) in cancer patients: a feasibility study. Proc. Ann. Meet. Am. Soc. Clin. Oncol. 12, 297 (1993). 
8. Herrero YCM, Cornelis JJ, Herold-Mende C Rommelaere J, Schlehofer JR, Geletneky K: Parvovirus $\mathrm{H}-1$ infection of human glioma cells leads to complete viral replication and efficient cell killing. Int. J. Cancer 109(1), 76-84 (2004).
9. Di Piazza M, Mader C, Geletneky K et al.: Cytosolic activation of cathepsins mediates parvovirus $\mathrm{H}-1$-induced killing of cisplatin and trail-resistant glioma cells. J. Virol. 81(8), 4186-4198 (2007).
10. Geletneky K, Kiprianova I, Ayache A et al.: Regression of advanced rat and human gliomas by local or systemic treatment with oncolytic parvovirus $\mathrm{H}-1$ in rat models. Neuro. Oncol. 12(8), 804-814 (2010). 